\title{
ODF-ANALYSIS OF MULTIPEAK TEXTURES
}

\author{
H. J. BUNGE, P. R. MORRIS and C. U. NAUER-GERHARDT $\dagger$ \\ Department of Physical Metallurgy, TU Clausthal, FRG.
}

(Received 19 January 1989)

\begin{abstract}
Multipeak textures are defined by an orientation distribution function consisting of a large number (e.g. 100) of narrow peaks (e.g. $1^{\circ}$ ) at the orientations $g^{i}$. Between these peaks the orientation density drops to zero. The peak orientations $g^{t}$ can be calculated from corresponding peak positions in one or several pole figures. Whether this procedure is unique or not depends on the number of peaks and the experimental uncertainty of the peak positions. The number of possible "ghost" positions may thus be estimated. A search procedure is described by which the orientations $g^{i}$ can be found from peak positions in three pole figures. The procedure was tested with several sets of randomly distributed orientations $g^{i}$.
\end{abstract}

KEY WORDS Multipeak textures, pole figure inversion, ghost orientations, resolving power, sphere rotation method.

\section{INTRODUCTION}

The texture of a polycrystalline material is described by the orientation distribution function (ODF) of its crystallites

$$
f(g)=\frac{d V / V}{d g}
$$

where $d V / V$ is the volume fraction of crystallites in the orientation $g$ within the limits $d g$.

The texture is a statistical quantity. This implies that the volume $d V$ in Eq. (1) consists of a sufficiently large number of crystallites. Hence, the volume element $d V$ must be replaced by a finite volume element $\Delta V$ which is large compared with grain size, and this in turn requires a finite angular element $\Delta g$ instead of $d g$ which thus defines a finite angular resolving power. The ratio of $\Delta V$ to grain size determines a statistical uncertainty $\pm \Delta f(g)$ of the distribution function $f(g)$. Under these premises, however, the distribution function $f(g)$ may have any shape, whatsoever.

Most of the texture functions, considered thus far, are "smooth" functions which do not exhibit strong variations within angular distances smaller than, say, 5 to 10 degrees. One of the sharpest preferred orientations was found in the cube recrystallisation texture which may have a half-maximum width in the range of $5^{\circ}$.

There are, however, also other examples where the texture function varies strongly over much shorter distances. One prominent example is the orientation distribution of a large number of small single crystal spheres sintered to a

† Now: Siemens AG, Karlsruhe 
monocrystalline substrate (see e.g. Maurer und Gleiter, 1986). By an appropriate heat treatment, these spheres may rotate to the nearest low-energy orientation. Hence, they assume a texture consisting of a certain number of very sharp peaks (of the order of $1^{\circ}$ ) each of which contains a different number of spheres. Orientation distribution functions of this type have been termed "multipeak" textures.

Although multipeak textures can, in principle, be treated with the general methods of texture analysis developed originally for "smooth" textures, just by increasing the resolving power, a more economic treatment may be obtained by using other methods specifically designed for this particular case. Such a specific method will be considered in the following.

\section{GENERAL PRINCIPLES OF TEXTURE ANALYSIS}

The analysis of multipeak textures is based on the same definitions, as introduced earlier, for the description of all kinds of textures, in general (Bunge, 1969). These general definitions will at first be summarized as far as they are needed for the specific considerations on multipeak textures given in the next section.

The orientation of an individual crystallite in a polycrystalline sample is defined by the rotation $g$ which transforms a sample coordinate system $K_{A}$ into a crystal coordinate system $K_{B}$

$$
K_{B}=g \cdot K_{A}
$$

The orientation $g$ can be expressed in many equivalent ways. In the following, three different representations will be used: i.e. the matrix representation $\left[g_{i j}\right]$; the representation by rotation axis $(\alpha \beta \gamma)$ and rotation angle $\omega$; representation by Euler angles $\varphi_{1} \phi \varphi_{2}$,

$$
g=\left[g_{i j}\right]=\{\alpha, \beta, \gamma, \omega\}=\left\{\varphi_{1}, \phi, \varphi_{2}\right\}
$$

The first of these representations is especially convenient when two or more rotations $g^{1}, g^{2}$ are to be carried out successively. Then the resulting rotation is given by the product

$$
g=g^{2} \cdot g^{1} ; \quad g_{i j}=g_{i k}^{2} \cdot g_{k j}^{1}
$$

The axis-angle representation is used, in the present context, in order to consider the orientation distance between neighbouring orientations. The representation by Euler angles, finally, is principally used to express the orientation distribution function, Eq. (1), by independent variables. In this case, the orientation element $d g$ has the form

$$
d g=\frac{\sin \phi}{8 \pi^{2}} d \varphi_{1} d \phi d \varphi_{2}
$$

The orientation distribution function is normalized in multiples of the random density

$$
\oint f(g) d g=f^{r} \equiv 1
$$

A crystal of the orientation $g$ can also be described by the symmetrically equivalent orientations $g^{\prime}$

$$
g^{\prime}=g^{c} \cdot g
$$


where $g^{c}$ are the crystal symmetry rotations, the number of which is $m^{c}$. Hence, the texture function must be invariant with respect to $g^{c}$

$$
f\left(g^{c} \cdot g\right)=f(g) \text { crystal symmetry }
$$

Besides this "individual invariance" the texture function may exhibit a "statistical invariance" which holds only for the aggregate of crystals as a whole (the sample symmetry)

$$
f\left(g \cdot g^{s}\right)=f(g) \quad \text { sample symmetry }
$$

This latter symmetry is not present in the case of multipeak textures.

A specific crystal direction $\mathbf{h}$ is defined in the crystal coordinate system $K_{B}$ by its components $h_{j}$ or by spherical polar coordinates $\theta \gamma$ which are related to each other by

$$
\begin{aligned}
h_{1} & =\sin \phi \cos \gamma \\
\mathbf{h}=h_{2} & =\sin \phi \sin \gamma \\
h_{3} & =\cos \phi
\end{aligned}
$$

Similarly, a sample direction $y$ is defined in the sample coordinate system $K_{A}$ by the components $y_{i}$ or by the spherical polar coordinates $\alpha \beta$

$$
\begin{aligned}
y_{1} & =\sin \alpha \cos \beta \\
\mathbf{y}=y_{2} & =\sin \alpha \sin \beta \\
y_{3} & =\cos \alpha
\end{aligned}
$$

The crystal direction $\mathbf{h}$ which is parallel to the sample direction $\mathbf{y}$ can be expressed by the crystal orientation $g$ in the form

$$
\mathbf{y}=g \cdot \mathbf{h} ; \quad y_{i}=g_{i j} \cdot h_{j}
$$

The distribution of a specific kind of crystal direction $\mathbf{h}$ in the sample coordinate system $K_{A}$, according to the orientation distribution of the crystallites, is called the h-pole figure.

$$
P_{\mathbf{h}}(\mathbf{y})=\frac{d V / V}{d \mathbf{y}}
$$

Thereby the solid angular element $d \mathbf{y}$ is expressed by

$$
d \mathbf{y}=\sin \alpha d \alpha d \beta
$$

The pole figure is normalized in multiples of the random density in the form

$$
\frac{1}{4 \pi} \oint P_{h}(y) d y=P_{h}^{r} \equiv 1
$$

The pole figure is related to the ODF by the fundamental equation

$$
P_{\mathbf{h}}(y)=\frac{1}{2 \pi} \int_{\mathbf{h} \| \mathbf{y}} f(g) d \psi
$$

where $\psi$ is the angle of rotation about the common direction $\mathbf{h} \| \mathbf{y}$.

Texture measurements can be carried out by individual orientation measurements, i.e. by determining the orientations $g^{i}$ of a sufficiently large number of crystallites. With Eq. (1) the orientation distribution can then be constructed, if 
the relative volumes $V^{t}$ of these crystallites have also been determined. It is to be emphasized that in the definition Eq. (1) the individual orientations $g^{i}$ (as well as their relative volumes $V^{i}$ ) are no longer being considered, once the distribution function $f(g)$ has been constructed. The information about the individual $g^{i}$ is then lost, and only a "population statistics" of the individuals is kept.

Most texture measurements are, however, based on pole figure measurements. The orientation distribution function $f(g)$ is then obtained by solving Eq. (16) which can be done by various methods based on one of four principles:

1. Discretization (biaxial-pole figure method, vector method)

2. Series expansion (harmonic method)

3. Integral transformation (inversion formula)

4. Probabilistic methods (Imhof method, WIMV-method)

The crystal direction $\mathbf{h}$ as well as the sample direction $\mathbf{y}$ are defined by vectors. In a polycrystal diffraction experiment the crystallographically equivalent directions $\{\mathbf{h}\}$ cannot be distinguished. Hence, the symmetrically superposed pole figures

$$
\tilde{P}_{\{\mathbf{h}\}}(\mathbf{y})=\frac{1}{2 m^{c}} \sum_{0^{c}} P_{0^{c} \cdot \mathbf{h}}(\mathbf{y})
$$

must be considered, where $0^{c}$ are all crystal symmetry operations and $2 m^{c}$ is the number of these symmetry operations. It has been shown (Bunge, Esling and Muller, 1981), that it is sufficient to consider only one symmetry operation of second kind in Eq. (17). In the case of centro-symmetric crystals, or by virtue of Friedel's law, this element can be chosen as the inversion centre (Matthies, 1979). In this most important case Eq. (17) can be reduced to only two terms

$$
\tilde{P}_{\{\mathbf{h}\}}(\mathbf{y})=\frac{1}{2}\left[P_{+\mathrm{h}}(\mathbf{y})+P_{-\mathbf{h}}(\mathbf{y})\right]
$$

Substituting $\tilde{P}_{\{\mathbf{h}\}}(\mathbf{y})$ in Eq. (16) for $P_{\mathbf{h}}(\mathbf{y})$ leads to a falsification of the obtained orientation distribution function, which is then termed the "reduced" ODF, $\tilde{f}(g)$ (Matthies, 1979). This ODF contains positive or negative "ghost peaks" (Lücke et al., 1981) which can, however, be reduced by taking the positivity condition of the ODF into account (Zero-range method-Bunge, Esling, 1979; positivity method-Dahms, Bunge, 1988). Nevertheless, the ambiguity introduced by replacing $P_{\mathrm{h}}(\mathbf{y})$ with $\tilde{P}_{\{\mathrm{h}\}}(\mathbf{y})$ can never by eliminated completely.

It should be mentioned, that this ambiguity problem does not arise in methods of ODF analysis based on individual orientations $g^{i}$. This applies equally well, whether the individual orientations $g^{i}$ appear as peaks in the final ODF as in the case of "multipeak textures," or they are being "smeared out" in order to construct a continuous "orientation density" function based on a large number of individual orientations $g^{i}$.

Texture analysis is usually carried out under the assumption of a reasonably "smooth" distribution function $f(g)$ with an angular resolving power of the order of magnitude of $5^{0}$ in the pole figures as well as in the orientation space. In the series expansion method this requires series expansion degrees of at least $L=22$. In the cubic orthorhombic case, this corresponds to 186 series terms and in the vector method, matrices of the size of $3240 \times 2592$ i.e. with 8398080 terms have been used. In order to obtain a resolving power of $1^{0}$, series expansions up to 
more than $L=100$ would be necessary whereby the number of coefficients increases proportional to $l^{3}$. In the vector method the number of terms would increase by a factor of $5^{5}$. Pole figure inversion corresponding to this resolving power can presently not be achieved with any of the existing methods.

It is the purpose of this paper to present a pole figure inversion method for the specific case of "multipeak" textures with a resolving power in the order of one degree or better.

\section{MULTI-PEAK TEXTURES}

We consider a specific type of orientation distribution function consisting of a large number $N$ (e.g. in the order of magnitude of 100 or more) of separate orientations $g^{i}$ with certain spread ranges $\Delta g^{i}$ about them (e.g. in the order of magnitude of $1^{0}$ or less) as is shown schematically in Figure 1a in a onedimensional example. The orientation distribution in the vicinity of the peak orientation $g^{i}$ may have different forms as is also shown in Figure 1a. The density drops, however, to zero within $\Delta g^{i}$ and is thus zero in the largest part of the orientation space.

a)

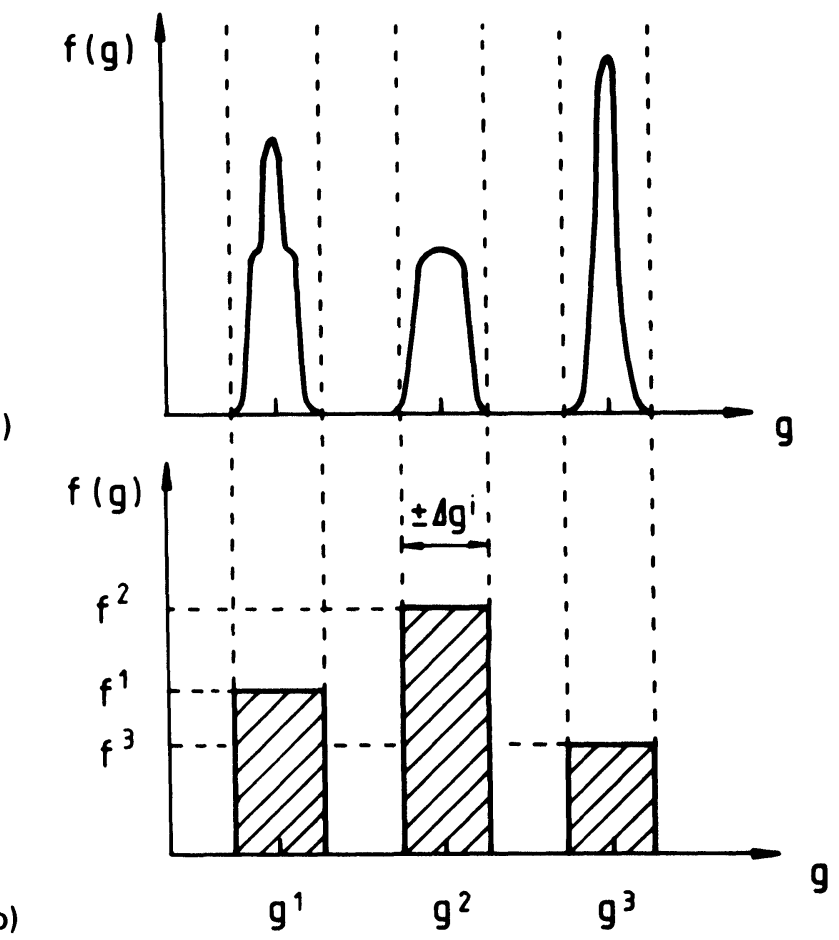

Figure 1 Schematic (one-dimensonal) representation of multipeak textures. a) The orientation density is zero except for a small environment $\Delta g^{i}$ of certain peak orientations $g^{i}$. The distribution function $f(g)$ may have different shapes in $\Delta g^{i}$. b) The shape of the distribution function within $\Delta g^{i}$ is not considered. 
Multipeak textures may be considered at two levels of experimental resolving power $\Delta_{\text {exp }}$ :

1) $\Delta_{\exp } \ll \Delta g^{i}$ high resolving power

2) $\Delta_{\exp } \approx \Delta g^{i}$ moderate resolving power

-In the first case, the exact shape of the distribution function $f(g)$ within each spread range $\Delta g^{i}$ is to be considered

- In the second case the exact distribution is not considered. Each orientation peak $g^{i}$ is then characterized by its central orientation $g^{i}$, by the spread range $\Delta g^{i}$ and by the integrated density $f^{i}$ as is shown schematically in Figure $1 \mathrm{~b}$.

In the present paper we shall consider only this second "level of resolving power." According to Eq. (7) each orientation $g^{i}$ has $m^{c}$ equivalent orientations $\mathrm{g}^{\prime i}$ in the whole orientation space. If we assume $N$ different orientations, each with a spread range $\Delta g$ defined according to Eq. (5) and knowing the integral density in the whole orientation space according to Eq. (6), the integrated density, mean density, and maximum density for an individual orientation $g^{i}$ can be estimated with the relationships

$$
\begin{gathered}
N \cdot m^{c} \cdot f^{i}=1 \\
f^{i}=f^{\text {mean }} \cdot \Delta g^{i} \\
f^{\text {max }}=a \cdot f^{\text {mean }}
\end{gathered}
$$

where " $a$ " is some "peak-shape factor." An example is given in Table 1. In the case of $2^{\circ}$ spread in each Euler angle and 100 orientations, the mean orientation density within each peak is thus in the order of magnitude of 1500 as compared to 15 as a usual value in smooth textures.

Each orientation $g^{i}$ gives rise to several peaks in the pole figures due to the crystallographically equivalent crystal directions

$$
\mathbf{h}^{j}=0^{j} \cdot \mathbf{h}
$$

Table 1 Characteristic values of a multipeak texture.

\begin{tabular}{|lll|}
\hline \multicolumn{1}{|c|}{ Number of orientations $g^{i}$} & $N$ & 100 \\
\hline Symmetrically equivalent orientations $g^{i}$ & $m^{c}$ & 24 \\
\hline $\begin{array}{l}\text { Spread range in } \\
\text { Euler angles } \\
\text { (Experimental uncertainty) }\end{array}$ & $\begin{array}{c}\Delta \varphi_{1} \\
\Delta \phi\end{array}$ & $\begin{array}{l}2^{\circ} \\
2^{\circ}\end{array}$ \\
\hline $\begin{array}{l}\text { Occupied volume element in the Euler } \\
\text { space for } \text { one orientation }\end{array}$ & $\Delta g^{i}$ & $\sim 4 \cdot 10^{-7}$ \\
\hline Total occupied volume in the Euler space & $\sum \Delta g^{i}$ & $\sim 10^{-5}$ \\
\hline $\begin{array}{l}\text { Mean orientation density within an } \\
\text { orientation } g^{i}\end{array}$ & $f^{\text {mean }}$ & $\sim 1550$ \\
\hline Maximum orientation density & $f^{\text {max }}$ & $\sim 8000$ \\
\hline
\end{tabular}


Thereby crystal symmetry rotations as well as inversions have to be considered. The number $m_{\mathbf{h}}$ of different directions $\mathbf{h}^{j}$ is equal to $2 \mathrm{~m}^{c}$ for non-enantiomorphic crystals and for general directions $\mathbf{h}$. For special directions the number $m_{\mathbf{h}}$ is a fraction thereof

$$
m_{\mathbf{h}}=\frac{2 m^{c}}{u_{\mathrm{h}}}
$$

where $u_{\mathbf{h}}$ is an integer which defines the "multiplicity" of the direction $\mathbf{h}$. (The symmetrically equivalent directions $\left\{\boldsymbol{h}^{\prime}\right\}$ are the same for the symmetrically equivalent orientations $g^{i}$ ). Hence, each orientation $g^{i}$ gives rise to $m_{\mathrm{h}}$ peaks in the h-pole figure at the positions

$$
\mathbf{y}_{\mathbf{h}}^{i j}=0^{j} \cdot g^{i} \cdot \mathbf{h}
$$

The peak widths are comparable to those in the orientation space

$$
\Delta y_{\mathrm{h}}^{i j} \approx \Delta g^{i}
$$

As in the orientation space, we do not distinguish the shape of the distribution function within each pole figure peak. Then the integrated density $P_{\mathrm{h}}^{i j}$ of a pole figure peak is related to the integrated density $f^{i}$ in Euler space by the relationship

$$
m_{\mathrm{h}} \cdot P_{\mathrm{h}}^{i j}=4 \pi \cdot m^{c} \cdot f^{i}
$$

This is illustrated in Figure 2. Thereby it must be kept in mind that pole figure peaks belonging to the same orientation $g^{i}$ (connected by dashed lines in Figure 2) cannot initially be distinguished from peaks belonging to all other crystal orientations. Hence, the peaks are numbered individually by the index $n$ which then replaces the two indices $i$ and $j$.

The main question which has to be answered is thus the question which values $n$ belong to which orientation $i$ used in Eq. (26).

It follows, of course, from Eq. (28) that pole figure peaks in the same pole figure belonging to the same orientation $i$ must have the same integrated density

$$
P_{\mathrm{h}}^{i j}=P_{\mathrm{h}}^{i j^{\prime}}
$$

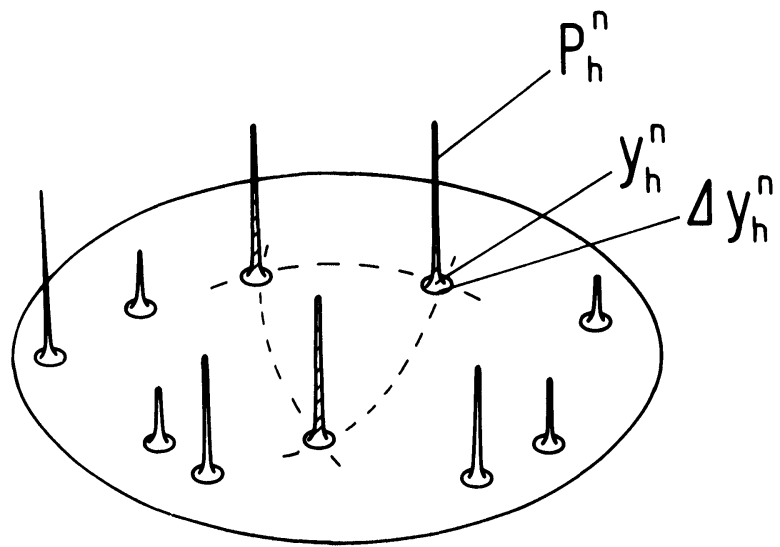

Figure 2 A pole figure consists of peaks of the integrated density $P_{\mathrm{h}}^{n}$ and the width $\Delta y_{\mathrm{h}}^{n}$ at the positions $\mathbf{y}_{\mathrm{h}}^{n}$. It is not known which peaks belong to one particular orientation $g^{i}$ (indicated by dashed lines). 
Peaks of the same orientation but in different pole figures are related to each other by

$$
m_{\mathbf{h}} \cdot P_{\mathbf{h}}^{i j}=m_{\mathbf{h}^{\prime}} \cdot P_{\mathbf{h}^{\prime \prime}}
$$

It must be mentioned in this connection that experimental pole figure measurements do not originally yield the normalized pole density values. The measured intensity then contains an unknown normalization factor $w_{\mathrm{h}}$ which depends on the experimental conditions and which is different for different crystal directions $\mathbf{h}$. Hence, the experimentally measured integrated intensity of one pole figure peak must be written

$$
I_{\mathrm{h}}^{i j}=w_{\mathrm{h}} \cdot P_{\mathrm{h}}^{i j}
$$

Hence, Eq. (29) comparing the pole densities of one orientation within one pole figure can be applied immediately to the measured intensities whereas Eq. (30) comparing peaks of the same orientation but within different pole figures can only be applied after normalization i.e. after $w_{h}$ is known.

Peaks $y_{h}^{i, j}$ in the pole figure belonging to different orientations $g^{i}$ may be superposed or they may be so close to each other that their integrated densities cannot be measured separately. The integrated density of such a superposed peak is the sum of the densities of all orientations $g^{i}$ contributing to it. In this case Eq. (28) must be generalized

$$
P_{\mathbf{h}}^{n}=4 \pi \frac{m^{c}}{m_{\mathbf{h}}} \sum_{i=1}^{i_{\max }} f^{i}
$$

The sum is to be extended over all orientations $i$ which contribute to the pole figure peak $n$. A relationship of the form of Eq. (32) is to be considered for all peaks in all pole figures whereby each peak may contain contributions of different orientations $i$. Then these intensity relationships can be summarized into one system containing all pole figure peaks and all orientations $g^{i}$

with

$$
I_{\mathbf{h}}^{n}=4 \pi \frac{m^{c}}{m_{\mathbf{h}}} w_{\mathrm{h}} \sum_{i=1}^{N} \lambda_{i}^{n} \cdot f^{i}
$$

$$
\begin{array}{ll}
\lambda_{i}^{n}=1 & \text { if } i \text { contributes to } n \\
=0 & \text { if } i \text { does not contribute to } n
\end{array}
$$

Thereby $N$ is the total number of orientations $g^{i}$.

The system of equations, Eq. (33), contains two types of unknowns, i.e. the normalization factors $w_{\mathrm{h}}$ of the various pole figures and the integrated densities $f^{i}$ of the orientations $g^{i}$. A most general solution of this system can be achieved iteratively:

In a first step certain approximations are assumed for the normalization factors $w_{\mathrm{h}}$ and the system is solved for the $f^{i}$.

In a second step the so obtained values $f^{i}$ are known and the system is solved for the $w_{\mathrm{h}}$ as unknowns.

These two steps are repeated until no further changes of the $w_{\mathrm{h}}$ and $f^{i}$ occur.

In practice, much easier special ways of solving Eq. (33) may be found: Since Eq. (33) is strongly overdetermined a solution may already be obtained from one pole figure. Then $w_{\mathrm{h}}$ is constant and can be obtained from the normalization condition Eq. (6).

A solution for the $w_{h}$ may also be obtained from one orientation $g^{i}$ if 
non-superposed pole figure peaks of this orientation can be found in each pole figure. This defines a subset of equations in Eq. (33), in which only one $\lambda_{i}^{n} \neq 0$ occurs in each equation. Hence, the relative values of $w_{\mathrm{h}}$ are thus obtained.

Later on, a search procedure will be considered which calculates the orientations $g^{i}$ from experimentally determined pole figure peaks $\mathbf{y}_{\mathbf{h}}^{n}$. In this procedure a tolerance $\varepsilon$ must be admitted which must be chosen slightly larger than the experimental uncertainty $\eta$ of the peak positions $\mathbf{y}_{h}^{n}$, in order to find all real orientations. As will be shown in the next section, this may give rise to "ghost" orientations found by the search procedure, which do not really exist. These ghost orientations can be sorted out by the integrated densities $f^{i}$. If a pole figure peak $\mathbf{y}_{\mathrm{h}}^{n}$ is due to real orientation $g^{i}$, and at the same time it is also allowed to belong to a certain ghost orientation $g^{j}$, then its density $P_{h}^{n}$ must correspond to the integrated intensity $f^{i}$ of the real orientation and the density of the ghost orientation must turn out to be zero. The differentiation between real and ghost peaks can thus be made by solving Eq. (33) which contains information about the same density $f^{i}$ in several pole figure densities $P_{\mathbf{h}}^{n}$.

Thereby "ghost" orientations can be distinguished from real ones by the condition

$$
f^{j}=0 \text { ghost }
$$

Hence, the procedure of ODF analysis for multipeak textures consists of two parts:

1. A search procedure which has to find all real $g^{i}$ (and possibly some ghost peaks)

2. The solution of Eq. (33) which gives the volume fractions $f^{i}$ of the orientations $g^{i}$ and excludes ghosts by the condition Eq. (35).

Multipeak textures of different types may be considered: Gleiter and coworkers developed a method to measure the orientation differences of low-energy grain boundaries by a sphere rotation method (see e.g. Maurer and Gleiter, 1986). Thereby some $10^{6}$ small single crystal spheres of $\sim 30 \mu \mathrm{m}$ diameter are sintered at first in random orientations onto a single crystalline substrate. During annealing at an appropriate temperature the spheres can rotate into the nearest low energy orientation corresponding to a cusp of the energy-misorientation function. It is not very well known how sharp these cusps are. However, the substructure of the substrate is often of the order of magnitude of one or two degrees. Hence, the final orientations of the spheres are found in peaks with a spread range of one or two degrees, just as assumed above, the integrated density corresponding to the number of spheres in this orientation.

Experimental measurements of multipeak pole figures can be carried out with a resolving power of the same order of magnitude using a scanning procedure with steps of one or two degrees.

A second problem where multipeak textures may be of interest is in studies of multicrystals i.e. samples consisting of a large number of crystallites which can, however, still be individually distinguished by their peaks in the pole figures as opposed to polycrystals where this is not the case. If the crystallites have a high degree of perfection, then the peak widths may be in the range $0.1^{\circ}$ to $0.01^{\circ}$. In this case, the peak width in the experimental measurements may be determined by the resolving power of the equipment used rather than by the natural peak 
width of the crystallites. Synchrotron radiation may then be valuable because of its highest resolving power among all diffraction methods. It is, however, to be mentioned that the scanning of a whole pole figure with a step width of $0.1^{\circ}$ or even $0.01^{\circ}$ is a serious problem which may require special considerations in order to reduce the measuring time.

\section{GHOST ORIENTATIONS}

According to Eq. (26) an orientation $g^{i}$ is determined from its pole figure peaks $\mathbf{y}_{\mathbf{h}}^{i, j}$. If the positions of these peaks were known with "mathematical" accuracy then the peaks belonging to $g^{i}$ could be identified uniquely independent of the presence of peaks belonging to other orientations $g^{k}$. If, however, the peak positions are known only with the "experimental" accuracy $\eta$, then a peak search procedure must accept peaks within a tolerance circle with the radius $\varepsilon$ around the ideal positions $y_{h}^{i, j}$, Figure 3 . In order not to lose an orientation, the tolerance circle $\varepsilon$ must be slightly larger than the uncertainty $\eta$. In this case, it may happen, with a certain probability, that pole figure peaks belonging to different orientations $g^{k}, g^{l}, g^{m} \ldots$ are, just by chance, in the right positions to fake an orientation $g^{i}$ (which really is not occupied in the texture). Such orientations shall be called "ghost orientations." The probability for any randomly chosen orientation $g^{i}$ to be a ghost orienation can easily be estimated.

A peak within a tolerance circle of the (small) radius $\varepsilon$ (in radians) about the correct position will be taken as belonging to the considered orientation $g^{i}$. The probability $p_{1}$ of finding one of the $m_{\mathrm{h}}$ peaks of the arbitrary orientation $g^{k}$ within the tolerance circle $\varepsilon$ is given by the ratio of the areas on the pole sphere, Figure 4

$$
p_{1}=\frac{\varepsilon^{2} \cdot \pi}{4 \pi / m_{\mathrm{h}}}=\frac{m_{\mathrm{h}} \cdot \varepsilon^{2}}{4}
$$

The probability for the circle to be empty of peaks is

$$
1-p_{1}=1-\frac{m_{\mathbf{h}} \cdot \varepsilon^{2}}{4}
$$

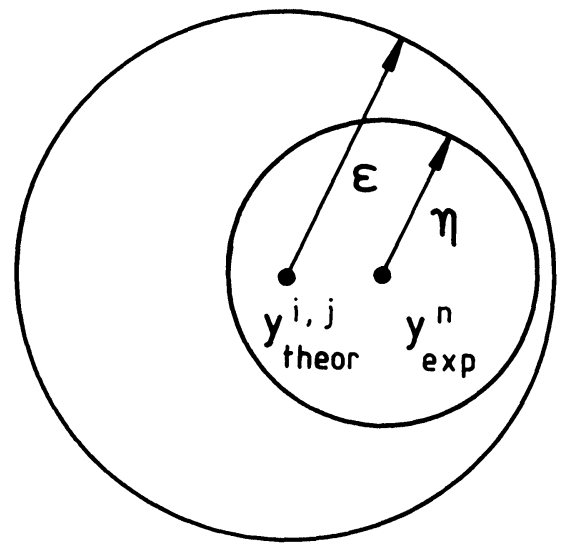

Figure 3 The uncertainty $\eta$ of experimentally determined peak positions $y_{\exp }^{n}$ and the tolerance circle $\varepsilon$ around a theoretical position $y_{\text {theor }}^{i j}$. 


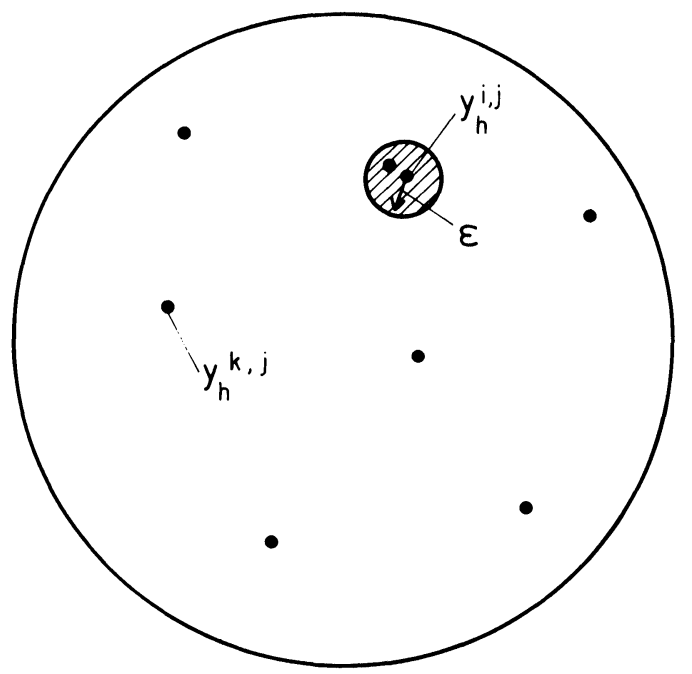

Figure 4 Probability of finding one of $m_{h}$ poles $y_{h}^{k, j}$ of an arbitrary orientation $g^{k}$ within the tolerance circle $\varepsilon$ around the pole $\mathbf{y}_{\mathbf{h}}^{i, j}$ of a considered orientation $g^{i}$.

If there are $N$ orientations, then the probability that none of them has a pole in the circle is given by the product of all these probabilities

$$
\left(1-p_{1}\right)^{N}=\left(1-\frac{m_{\mathrm{h}} \cdot \varepsilon^{2}}{4}\right)^{N}
$$

The probability for finding at least one peak in the circle is

$$
1-\left(1-p_{1}\right)^{N}=1-\left(1-\frac{m_{\mathbf{h}} \cdot \varepsilon^{2}}{4}\right)^{N}
$$

The ghost probability, finally, is the probability of finding at least one peak in the tolerance circles of each pole used to identify the orientation $g^{i}$. It is assumed that $m_{h}^{\prime}$ poles in the h-pole figure are considered. Then the probability for an arbitrary orientation to be accepted as a possible orientation (ghost probability) is given by

$$
p_{\text {ghost }}=\Pi_{\mathbf{h}}\left[1-\left(1-\frac{m_{\mathbf{h}} \cdot \varepsilon^{2}}{4}\right)^{N}\right]^{m_{\mathbf{h}}}
$$

As long as $\varepsilon$ is small enough this probability can be approximated by

$$
p_{\text {ghost }} \approx \Pi_{\mathbf{h}}\left[\frac{1}{4} N m_{\mathbf{h}} \varepsilon^{2}\right]^{m_{\mathbf{h}}}=\left[\frac{1}{4} N \varepsilon^{2}\right]^{\sum m_{\mathbf{h}}} \cdot \Pi_{\mathbf{h}} m_{\mathbf{h}}^{m_{\mathbf{h}}}
$$

with the values of Table 2 this can be written

$$
p_{\text {ghost }} \approx N^{7} \cdot \varepsilon^{14} \cdot 243
$$

If we admit a certain ghost probability $p_{\max }$ as tolerable then the tolerance $\varepsilon$ needed in order to safely distinguish $N$ random orientations in a multipeak 
Table 2 Number of peaks in different pole figures and deduced quantities.

\begin{tabular}{|l|c|c|r|}
\hline & $(111)$ & $(200)$ & $(220)$ \\
\hline$m_{\mathbf{h}}$ & 8 & 6 & 12 \\
\hline$m_{\mathbf{h}}^{\prime}$ & 2 & 2 & 3 \\
\hline$m_{\mathbf{h}}^{m_{\mathbf{h}}}$ & 64 & 36 & 1728 \\
\hline$\sum_{\mathbf{h}} m_{\mathbf{h}}$ & & 7 \\
\hline$\prod_{\mathbf{h}} m_{\mathbf{h}}^{m_{\mathbf{h}}}$ & \multicolumn{3}{|c|}{3981312} \\
\hline
\end{tabular}

texture is given by

$$
\varepsilon=\left(\frac{p_{\max }}{243}\right)^{1 / 14} \cdot \frac{1}{\sqrt{N}}
$$

This is shown in Figure 5 for $p_{\max }=10^{-3}$ and $\varepsilon$ expressed in degrees ("resolved" and "not-resolved" area). If, finally, an orientation search procedure is based on $M$ pairs of peaks in the (110) pole figure, which define two possible orientations, each, then the total number of ghost peaks can be estimated by

$$
N_{\text {ghost }}=2 M \cdot p_{\text {ghost }}
$$

According to Figure 8 the number of pairs having an acceptable distance from each other depends on the total number of peaks in the pole figure and on the angular tolerance $\varepsilon$. It is approximately

$$
M \approx\left(m_{\mathbf{h}}^{\prime} \cdot N\right)^{2} \cdot a \cdot \varepsilon
$$

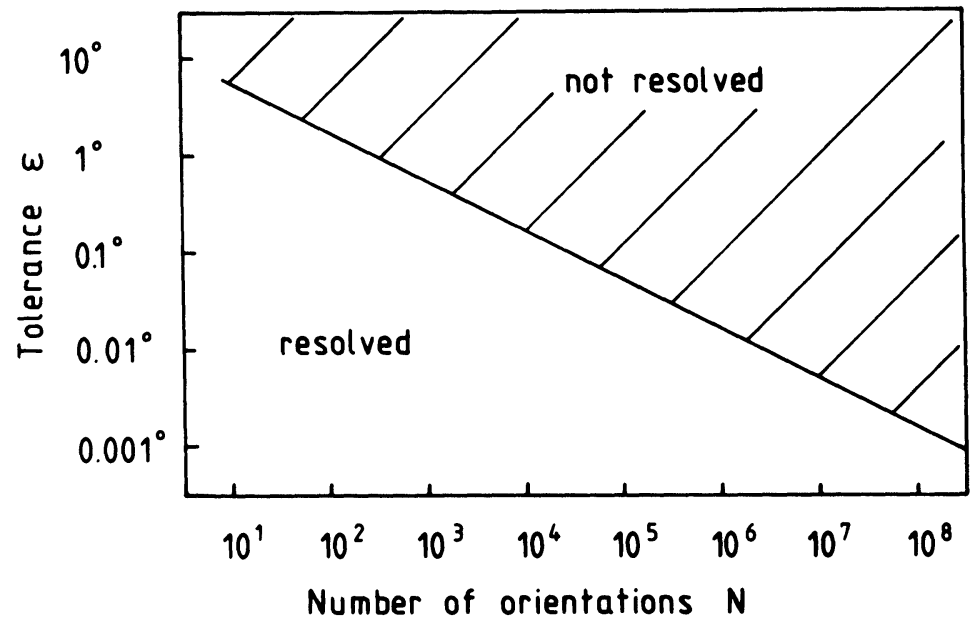

Figure 5 The resolved and not resolved region defined by a maximum ghost probability $p_{\max }=10^{-3}$ according to Eq. (43). 
where " $a$ " is a geometrical factor in the order of one. Hence, the total expected number of ghosts may be expressed

$$
N_{\text {ghost }} \approx 4400 \cdot N^{9} \cdot \varepsilon^{15}
$$

The extremely strong dependence of the expected number of ghosts on the admitted tolerance $\varepsilon$ shows that this parameter must be chosen very carefully.

With a resolving power of $1^{\circ}$, and $p_{\max }=10^{-3}$ as assumed above, it should thus be possible to determine 1500 individual orientations. By increasing the resolving power to $0.1^{\circ}$ or $0.01^{\circ}$ respectively, as could be realized by synchrotron radiation, the possible number of orientations increases to $1.5 \cdot 10^{5}$ or $1.5 \cdot 10^{7}$ respectively.

The estimation of the probability $p_{\text {ghost }}$ and hence of the number $N$ of orientations which can be determined, depends on the assumption of random distribution of the orientations $g^{i}$. If there is a preferred orientation of the crystallites then the orientations $g^{i}$ are closer to each other in certain ranges of the pole figures. This increases the probability of "ghost" orientations in these regions and hence decreases the number $N$ of orientations which can be safely separated.

\section{A SEARCH PROCEDURE}

In Figure 2 pole figure peaks in the positions $\mathbf{y}_{\mathbf{h}}^{n}$ are shown, some of which belong to the same orientation $g^{i}$. It is, however, not known at the beginning, which ones these are. Hence, a search procedure has to be used by which it can be decided whether other peaks $\mathbf{y}_{\mathbf{h}^{\prime}}$ ' may belong to the same orientation $g^{i}$ as the peak $\mathbf{y}_{\mathbf{h}}^{n}$, and if all peaks of the orientation $g^{i}$ are really found. This question cannot be answered mathematically exactly because of the uncertainty $\eta$ of the peak positions in the pole figures. If the natural peak width is $\Delta \mathbf{y}_{\mathbf{h}}^{i, j}$ and the experimental resolving power is $\Delta_{\text {exp }}$ then the uncertainty $\eta$ of any peak position in the pole figure is of the order of magnitude

as is shown in Figure 6.

$$
\eta \approx \Delta \mathbf{y}_{\mathbf{h}}^{i j}+\Delta_{\text {exp }}
$$

A search procedure must be able to find any orientation $g^{i}$. In order to identify an orientation $g^{i}$ by its poles $y_{\mathrm{h}}^{i j}$ in one or several pole figures, at least two poles are needed (if the connecting plane of the poles is a mirror plane). If this is not the case then an ambiguity of two possible orientations remains which can only be removed by looking for a third pole on either side of the connecting plane. Hence, if one wants to identify an orientation by poles in one pole figure, then two neighbouring poles are enough in the (100) and (111) pole figures, for instance, but three are needed in the (110) pole figure, as is shown in Figure 7. It is assumed that pole figures can only be measured in the back-reflection range. It is then necessary to consider a range $B_{h}$ of the pole figure with the radius $\alpha_{h}$ chosen in such a way that at least two (or three) poles of any orientation are contained in the range $B_{\mathrm{h}}$. These ranges are given in Table 3 for the first three pole figures of the fcc-case.

We chose the (110) pole figure to start with. Then each peak $\mathbf{y}_{(110)}^{n}$ in this pole figure is taken as a starting peak. A second peak belonging to the same orientation must be found in a distance of $60^{\circ}$ as is shown in Figure (8). Because 


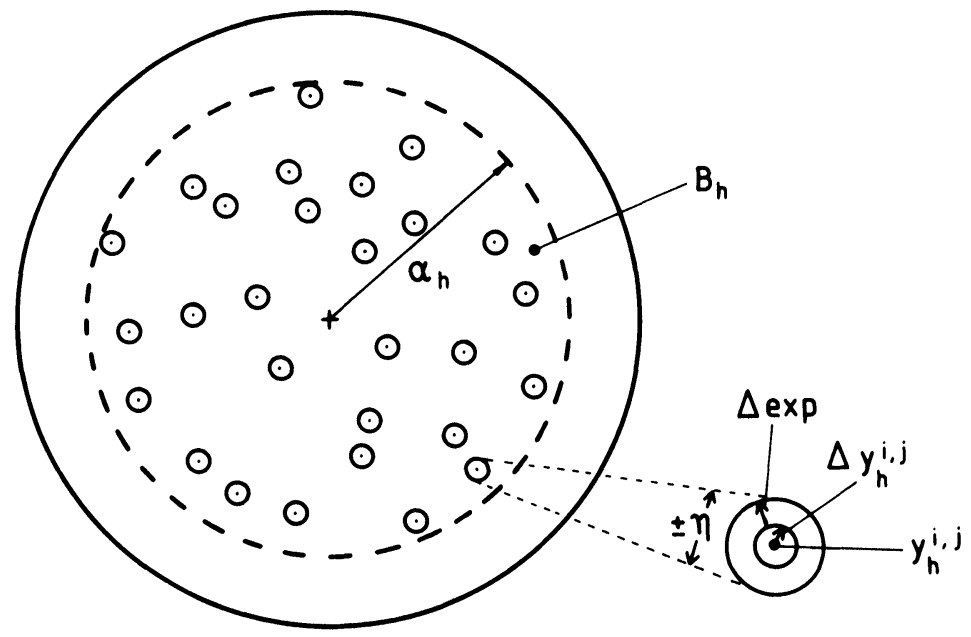

Figure 6 In the back-reflection range $B_{h}$ defined by $\alpha \leq \alpha_{h}$, pole figure peaks at the positions $y_{h}^{i, j}$ are measured. The uncertainty $\eta$ of the peak position is due to the natural peak width $\Delta \mathbf{y}_{\mathbf{h}}^{i, j}$ and the experimental error $\Delta_{\text {exp }}$.
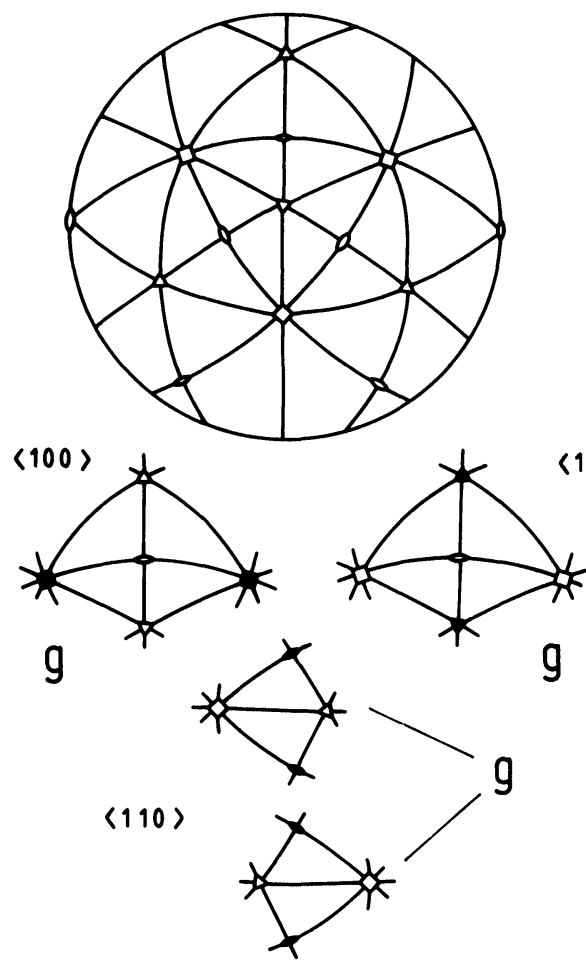

$\langle 111\rangle$

Figure 7 An orientation $g$ is fixed uniquely by two neighbouring $\langle 100\rangle$ or $\langle 111\rangle$ poles which are connected by a mirror plane but not by two $\langle 110\rangle$ poles, which are not connected by a mirror plane. 
Table 3 Radius $\alpha$ needed in order to identify any orientation by its poles in a pole figure.

\begin{tabular}{|lccc|}
\hline Pole figure & $(111)$ & $(200)$ & $(220)$ \\
\hline Maximum radius $\alpha_{\mathrm{h}}$ & $70.5^{\circ}$ & $90^{\circ}$ & $60^{\circ}$ \\
\hline
\end{tabular}

of the experimental uncertainty of each peak, all peaks within a band of the width $\pm \varepsilon$ must be considered to be possible counter parts (peak 2) to the peak 1 in Figure 8. In the (110) pole figure, this leaves the ambiguity of two possible orientations which are in mirror position with respect to the plane $(1,2)$. Hence, a third peak must be found either in the position 3 or $3^{\prime}$ within the limit $\varepsilon$. If

$$
\varepsilon>\eta
$$

then this procedure must find the correct orientation $g^{i}$. It may, however, happen that it also finds other points 2,3 , Figure 8 , which are in the right position just by chance and do not really belong to the same orientation $i$ as peak 1 . This was already called a ghost orientation. The probability of finding a ghost orientation can be reduced by looking for further poles of the same orientation within the measured range $B_{\mathrm{h}}$ of the (110) pole figure and in other pole figures i.e. the (200) and (111) pole figures in the present case. Only if all required poles are really found, an orientation is accepted for being a possible orientation.

Since the range $B_{(110)}$ was chosen in such a way that it contains at least three poles of any orientation, each orientation must thus be found at least three times. Because of the allowed tolerance $\varepsilon$ these three orientations are not exactly identical. They are only close to each other within the tolerance $\varepsilon$.

The search procedure starts with one of the experimental peak positions $\mathbf{y}_{\exp }^{n}$

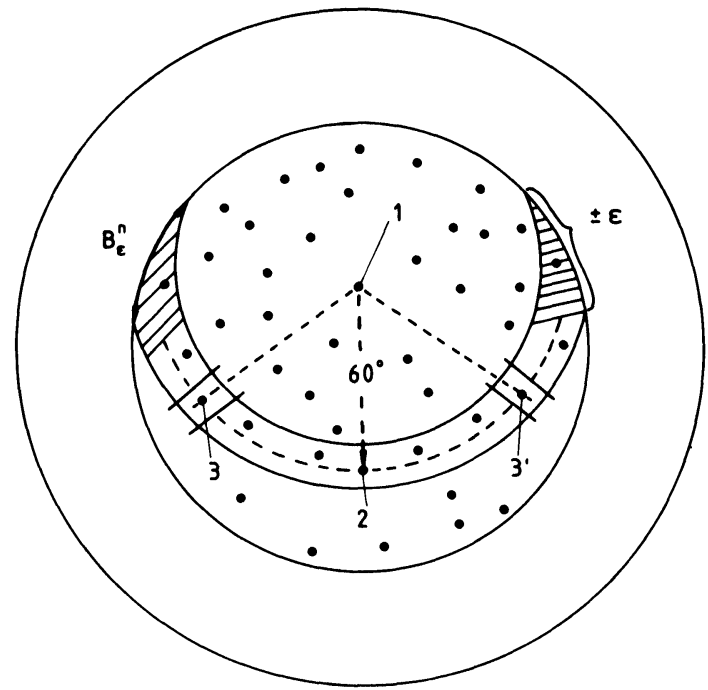

Figure 8 Poles in a (110) pole figure. Pole 1 is chosen as the starting pole. Pole 2 is found in a distance of $60^{\circ} \pm \varepsilon$. A third pole must be found either at 3 or $3^{\prime}$. 


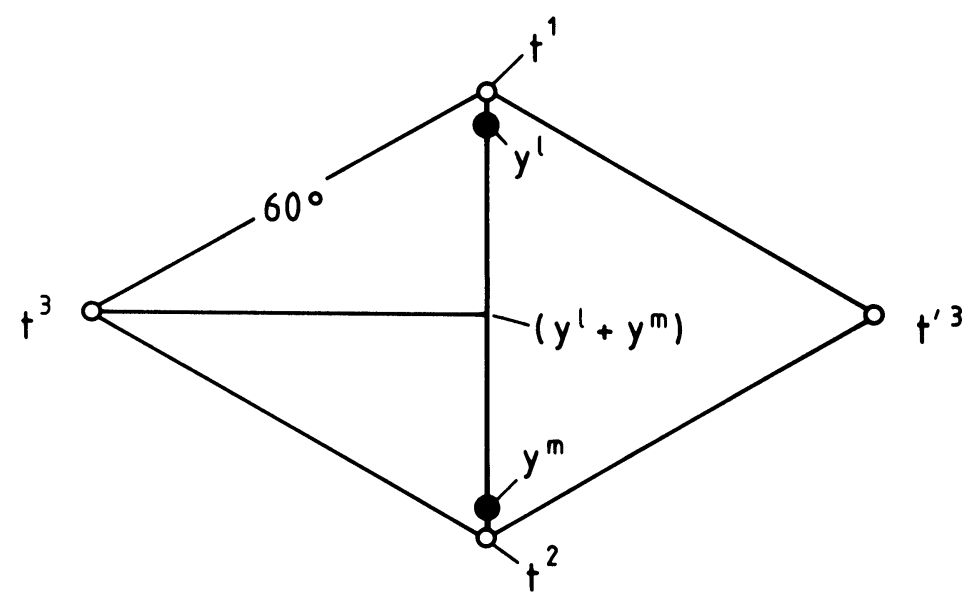

Figure 9 The experimental poles $y^{l}$ and $y^{m}$ and two sets of theoretical poles $\mathbf{t}^{1} \mathbf{t}^{2} \mathbf{t}^{3}$ and $\mathbf{t}^{1} \mathbf{t}^{2} \mathbf{t}^{3}$.

$\left(1 \leq n \leq N_{h}\right)$ of the (110) pole figure within $B_{(110)}$. It deviates from its "true" positions $\mathbf{y}_{\text {true }}^{n}$ by no more than

$$
\mathcal{4}\left(\mathbf{y}_{\exp }^{n}, \mathbf{y}_{\text {true }}^{n}\right) \leq \eta
$$

Let the first chosen peak be $\mathbf{y}^{l}$. Then a second one $\mathbf{y}^{m}$ is found which fulfills the condition

$$
\cos \left(60^{\circ}+\varepsilon\right) \leq \mathbf{y}^{l} \cdot \mathbf{y}^{m} \leq \cos \left(60^{\circ}-\varepsilon\right)
$$

All possible peaks fulfilling this condition are considered for $\mathbf{y}^{m}$ with the same $\mathbf{y}^{1}$. Because of the experimental inaccuracy, $y^{l}$ and $\mathbf{y}^{m}$ do not have the correct distance of $60^{\circ}$. Hence two other points $\mathbf{t}^{1}$ in the vicinity of $\mathbf{y}^{l}$ and $\mathbf{t}^{2}$ in the vicinity of $\mathbf{y}^{m}$ are first constructed which have the correct distance. These points are in the connecting plane of $\mathbf{y}^{l}$ and $\mathbf{y}^{m}$ and have the same small distance from $\mathbf{y}^{l}$ and $\mathbf{y}^{m}$ respectively as shown in Figure 9. A third point $\mathbf{t}^{3}$ or $\mathbf{t}^{\prime 3}$ respectively is then found which has the correct distance of $60^{\circ}$ from $\mathbf{t}^{1}$ and $\mathbf{t}^{2}$ respectively on either side of the plane $\left(\mathbf{t}^{1}, \mathbf{t}^{2}\right)$, Figure 9 . These points are given by the vectors

$$
\begin{aligned}
\mathbf{t}^{1} & =\frac{\sqrt{3}}{2} \cdot \frac{\mathbf{y}^{l}+\mathbf{y}^{m}}{\left|\mathbf{y}^{l}+\mathbf{y}^{m}\right|}+\frac{1}{2} \frac{\mathbf{y}^{l}-\mathbf{y}^{m}}{\left|\mathbf{y}^{l}-\mathbf{y}^{m}\right|} \sim[110] ;[011] \\
\mathbf{t}^{2} & =\frac{\sqrt{3}}{2} \cdot \frac{\mathbf{y}^{l}+\mathbf{y}^{m}}{\left|\mathbf{y}^{l}+\mathbf{y}^{m}\right|}-\frac{1}{2} \frac{\mathbf{y}^{l}-\mathbf{y}^{m}}{\left|\mathbf{y}^{l}-\mathbf{y}^{m}\right|} \sim[011] ;[110] \\
\mathbf{t}^{3} & =\frac{\sqrt{3}}{2} \cdot \frac{\mathbf{y}^{l} \times \mathbf{y}^{m}}{\left|\mathbf{y}^{l} \times \mathbf{y}^{m}\right|}+\frac{1}{2} \frac{\mathbf{y}^{l}+\mathbf{y}^{m}}{\left|\mathbf{y}^{l}+\mathbf{y}^{m}\right|} \sim[101] ; \\
\mathbf{t}^{3} & =\frac{\sqrt{3}}{2} \cdot \frac{\mathbf{y}^{l} \times \mathbf{y}^{m}}{\left|\mathbf{y}^{l} \times \mathbf{y}^{m}\right|}+\frac{1}{2} \frac{\mathbf{y}^{l}+\mathbf{y}^{m}}{\left|\mathbf{y}^{l}+\mathbf{y}^{m}\right|} \sim
\end{aligned}
$$

The two choices of triples $\left(\mathbf{t}^{1}, \mathbf{t}^{2}, \mathbf{t}^{3}\right)$ and $\left(\mathbf{t}^{1}, \mathbf{t}^{2}, \mathbf{t}^{3}\right)$ fulfill the conditions

$$
\mathbf{t}^{1} \times \mathbf{t}^{2} \cdot \mathbf{t}^{3}>0 ; \quad \mathbf{t}^{1} \times \mathbf{t}^{2} \cdot \mathbf{t}^{3}<0
$$


Table 4 Elements of the transformation matrices $\kappa$ and $\kappa^{\prime}$.

$$
[\kappa]=\frac{1}{\sqrt{2}}\left[\begin{array}{lll}
1 & 1 & \overline{1} \\
\overline{1} & 1 & 1 \\
1 & \overline{1} & 1
\end{array}\right] ; \quad\left[\kappa^{\prime}\right]=\frac{1}{\sqrt{2}}\left[\begin{array}{lll}
\overline{1} & 1 & 1 \\
1 & 1 & \overline{1} \\
1 & \overline{1} & 1
\end{array}\right]
$$

In the sequence $\mathbf{t}^{1} \mathbf{t}^{2} \mathbf{t}^{3}$ or $\mathbf{t}^{1} \mathbf{t}^{2} \mathbf{t}^{3}$ they either form a right or left handed set which may be identified with the three $\langle 110\rangle$ directions as given in Eq. (51). The vectors $\mathbf{t}^{1}, \mathbf{t}^{2}, \mathbf{t}^{3}$ or $\mathbf{t}^{1}, \mathbf{t}^{2}, \mathbf{t}^{\prime 3}$ respectively form a non-orthogonal crystal coordinate system $K_{B}^{\prime}$ which fixes the crystal orientation $g$. The orientation matrix $\left[g_{i j}\right]$ is thus obtained by the transformation to an orthogonal crystal coordinate system $K_{B}$ used in Eq. (2).

$$
g_{i j}=t_{k}^{i} \cdot \kappa_{k j} ; \quad g_{i j}^{\prime}=t_{k}^{\prime i} \cdot \kappa_{k j}^{\prime}
$$

with the transformation matrices $\kappa_{k j}$ and $\kappa_{k j}^{\prime}$ given in Table 4.

For either of the orientations $g$ and $g^{\prime}$ the positions $\mathbf{y}_{\langle 110\rangle}$ of all $\langle 110\rangle$ directions in the (110) pole figure are then calculated using Eq. (12) and Eq. (7). Those values which are outside the experimental range $B_{(110)}$ are omitted. The other ones are checked with all experimental values $\mathbf{y}_{\exp }^{n}$ of this pole figure according to the condition

$$
\left|\mathbf{y}_{\langle 110\rangle} \cdot \mathbf{y}_{\exp }^{n}\right| \geq \cos \varepsilon
$$

The orientation $g$ or $g^{\prime}$ is accepted and stored in file if all calculated values $\mathbf{y}_{\langle 110\rangle}$ have found an experimental counterpart $\mathbf{y}_{\text {exp }}^{n}$ according to Eq. (54).

This procedure is repeated with all $\mathbf{y}^{m}$ for the same $\mathbf{y}^{l}$ and then for all values $\mathbf{y}^{l}$.

Each time a possible orientation $g$ or $g^{\prime}$ is found, it must be checked if a symmetrically equivalent orientation is already among the ones found previously. Thereby all cubic symmetry variants $g^{c} \cdot g$ of $g$ according to Eq. (7) have to be considered. The misorientation $\delta g$ between a symmetry variant of $g$ and a formerly found orientation $g^{n}$ is given by

$$
\delta g=g^{n^{-1}} \cdot g^{c} \cdot g
$$

The misorientation angle $\omega$ according to Eq. (3) is given by the trace of the misorientation matrix $\delta g_{i k}$

$$
\cos \omega=\frac{1}{2}(\operatorname{tr}|\delta g|-1)
$$

The two orientations $g$ and $g^{n}$ are assumed to be equal if

$$
\omega \leq \varepsilon
$$

If the two orientations are assumed to be equal then they have to be averaged. Thereby it may happen that the orientation $g^{n}$ has already been averaged once or even several times. This orientation is then given a higher weight in the average. The averaging is carried out by rotating the formerly found orientation $g_{i}^{n}$ by the rotation matrix $\delta g^{\prime}$ the rotation angle $\omega^{\prime}$ of which is a fraction of the angle $\omega \mathrm{Eq}$. (56)

$$
\omega^{\prime}=\frac{\omega}{i+1}
$$




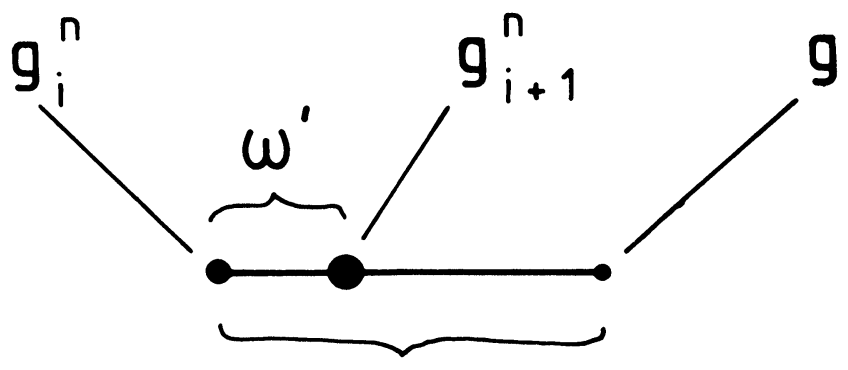

$\omega$

Figure 10 The orientation $g$ found in the distance $\omega$ from the previously found orientation $g_{i}^{n}$ is averaged with the latter one, taking the weight $i$ into account.

Thereby $i$ is the weight of the formerly obtained orientation. The averaged orientation is then

$$
g_{i+1}^{n}=\delta g^{\prime} \cdot g_{i}^{n}
$$

This is illustrated in Figure 10. The rotation matrix $\delta g^{\prime}$ can be obtained by expressing the matrix $\delta g$ in terms of the rotation axis and angle, Eq. (3), in the form

$$
\delta g=\left[\begin{array}{ccc}
\sin ^{2} \alpha \cdot \cos \omega+\cos ^{2} \alpha & \cos \alpha \cdot \cos \beta \cdot(1-\cos \omega) & \cos \alpha \cdot \cos \gamma \cdot(1-\cos \omega) \\
\cos \alpha \cdot \cos \beta \cdot(1-\cos \omega) & \sin ^{2} \beta \cdot \cos \omega+\cos ^{2} \beta & -\cos \beta \cdot \cos \gamma \cdot(1-\cos \omega) \\
-\cos \gamma \cdot \sin \omega & & +\cos \alpha \cdot \sin \omega \\
\cos \alpha \cdot \cos \gamma \cdot(1-\cos \omega) & \cos \beta \cdot \cos \gamma \cdot(1-\cos \omega) & \sin ^{2} \gamma \cdot \cos \omega+\cos ^{2} \gamma \\
+\cos \beta \cdot \sin \omega & -\cos \alpha \cdot \sin \omega &
\end{array}\right]
$$

The new orientation $g_{i+1}^{n}$ then replaces the old one $g_{i}^{n}$ in file.

The averaging procedure Eq. (59) changes the position of a formerly found orientation. Hence, it may happen that orientations which were formerly considered to be different with respect to the condition Eq. (57) have now become equal. Hence, all obtained orientations must finally be checked again with Eq. (57) and averaged, if necessary.

Finally, the $g^{n}$-values having passed this procedure are checked with the poles in the (111) and (200) pole figures inside the experimental ranges $B_{\mathrm{h}}$ using Eq. (12) with $\mathbf{h}=[111]$ and $\mathbf{h}=$ [200] respectively. Orientations $\boldsymbol{g}^{n}$ having passed this check, too, are finally accepted as possible $g^{i}$-values.

With the orientations $g^{t}$ known, the pole figure peaks $\mathbf{y}_{\mathbf{h}}^{n}$ can be identified with poles $\mathbf{y}_{\mathbf{h}}^{i, j}$ belonging to an orientation $g^{i}$

$$
\mathbf{y}_{\mathbf{h}}^{i j} \rightarrow \mathbf{y}_{\mathbf{h}}^{n}
$$

In order not to loose a really existing orientation, a tolerance $\varepsilon$ had to be admitted in the search procedure which was slightly larger than the experimental 
uncertainty $\eta$ (see Eq. (48)). Hence, the resolving power of the search procedure in the $g$-space (defined by Eq. (57)) is slightly smaller than that in the pole figures. It may thus happen that two or more nearby orientations $g^{i_{1}}, g^{i_{2}} \ldots$ are not resolved in $g$-space although their pole figure peaks are separated. If this has happened, then several distinct pole figure peaks $\mathbf{y}_{\mathbf{h}}^{n_{1}}, \mathbf{y}_{\mathbf{h}}^{n_{2}} \ldots$ are identified in Eq. (61) with the same calculated pole $y_{h}^{i, j}$. Hence, Eq. (61) has to be generalized in the form

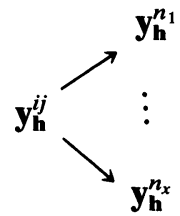

In this case the intensities of the corresponding pole figure peaks must be added

$$
I_{\mathbf{h}}^{n}=\sum_{x} I_{\mathbf{h}}^{n_{x}}
$$

The total intensity $I_{\mathbf{h}}^{n x}$ then enters Eq. (33).

\section{NUMERICAL TEST OF THE METHOD}

The search procedure was tested numerically using different numbers of orientations $g^{i}$ chosen at random in the whole orientation space. The corresponding poles in the (100) (110) and (111) pole figure were then calculated using Eq. (12). In order to simulate the experimental situation the poles were shifted at random by $|\eta| \leq 1^{\circ}$ The result is shown in Figure 11. It is not possible to visualize

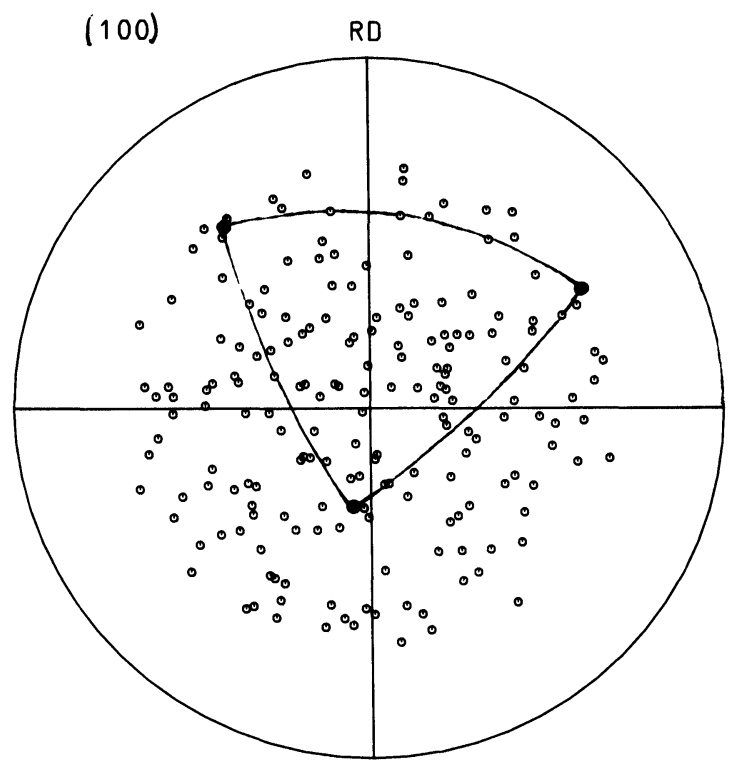

(a)

Figure 11 (a) The (100) poles of 100 randomly chosen orientations shifted by $|\eta| \leq 1^{\circ}$. The poles belonging to one particular orientation are marked. 


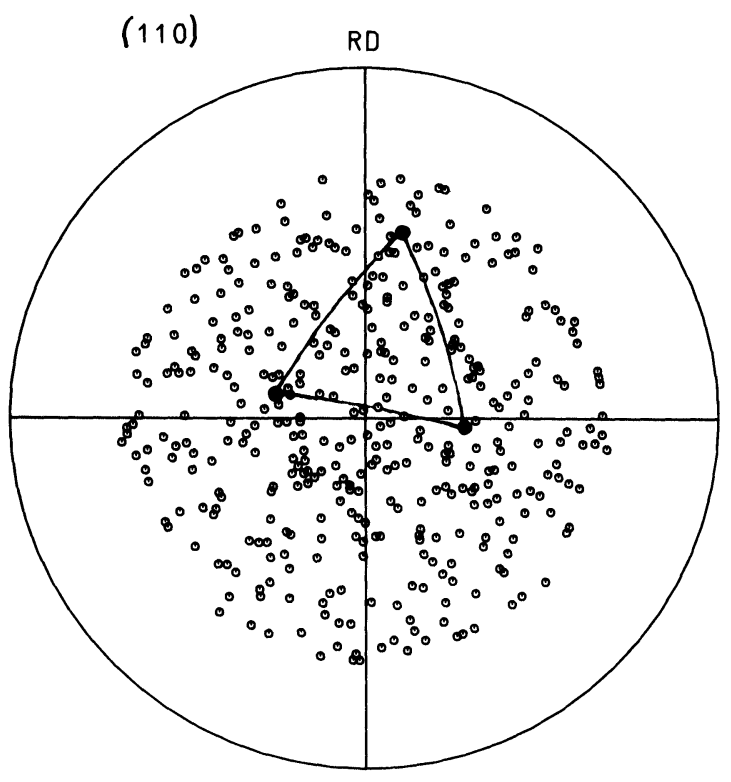

(b)

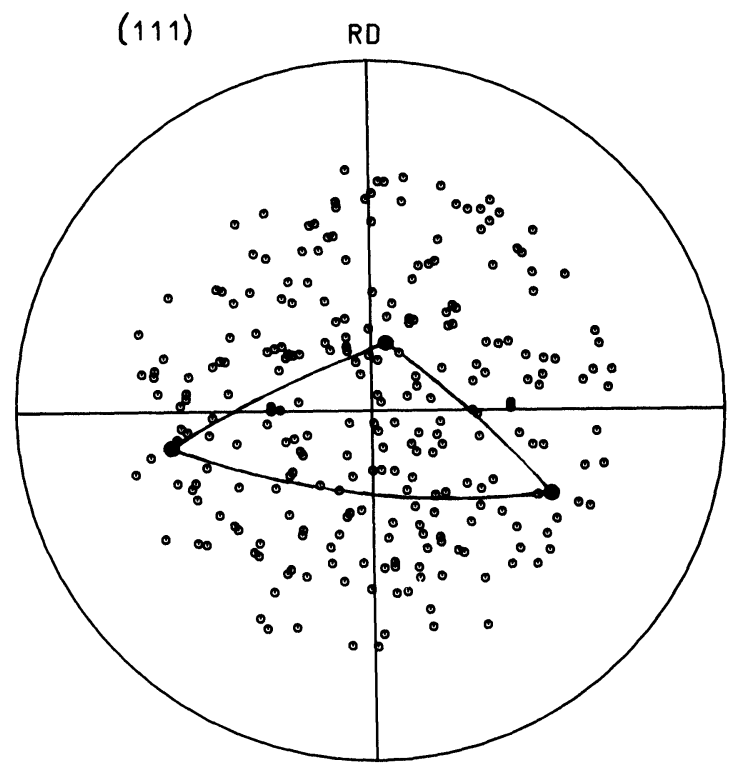

(c)

Figure 11 (b) and (c) The (110) (111) poles of 100 randomly chosen orientations shifted by $|n| \leq 1^{\circ}$. The poles belonging to one particular orientation are marked. 


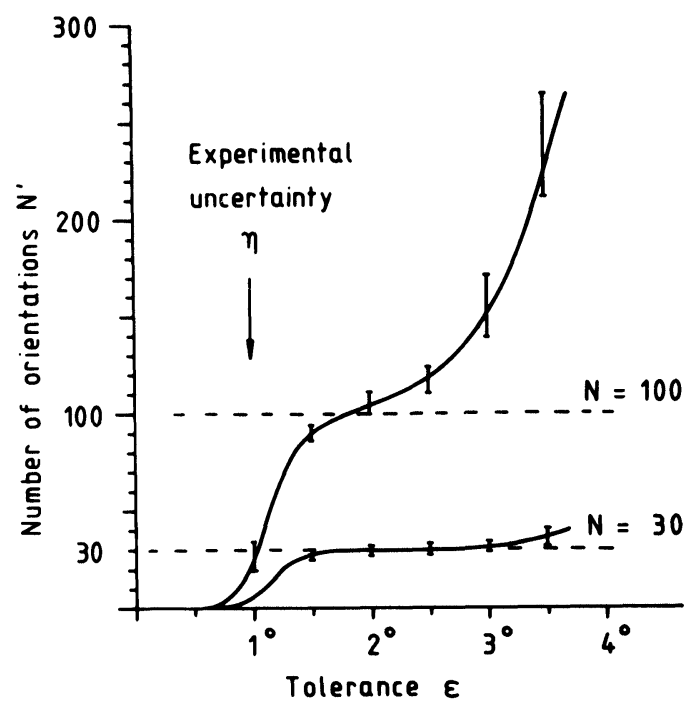

Figure 12 Number of orientations $N^{\prime}$ found by the search procedure as a function of the admitted tolerance $\varepsilon$. The uncertainty of the poles was $|\eta| \leq 1^{\circ}$. The error margins are obtained from different choices of $N=30$ and $N=100$ random orientations.

which poles belong to the same orientation. To serve as an example, the poles of one particular orientation are marked in Figure 11. The search procedure was then applied with different tolerances $\varepsilon$. Figure 12 gives the number $N^{\prime}$ of orientations found by the search procedure for two values of $N$ i.e. $N=30$ and $N=100$. The different values of $N^{\prime}$ for the same $\varepsilon$-value are due to different choices of $N$ random orientations as input.

It is seen that not all of the input orientations are found with a tolerance $\varepsilon$ equal to the uncertainty $\eta$ of the pole figure peaks. In fact, $\varepsilon$ must be chosen slightly larger than $\eta$. With $\eta=1^{\circ}$, a value of $\varepsilon=1.7^{\circ}$ is found to be sufficient. For $N=30$ this value of $\varepsilon$ is well within the "resolved" area of Figure 5. Even up to $\varepsilon=3^{\circ}$ the number of found orientations $N^{\prime}$ is very close to the number $N=30$ of input orientations. Only at $\varepsilon=3.5^{\circ}$ an increase is observed due to an increased number of ghost orientations. With $N=100$, the value $\varepsilon=1.7^{\circ}$ is already at the border of the resolved area. Hence, the curve already increases for values larger than $1.7^{\circ}$. With an experimental uncertainty $\eta=1^{\circ}$, the number of $N=100$ orientation is in this case the maximum number which can be resolved with a reasonably small number of ghost orientations.

\section{CONCLUSIONS}

A search procedure was developed which allows one to identify $N$ orientations $g^{i}$ in the orientation space from their poles in the back-reflection range of the (220) pole figure taking also into account the poles in the (111) and (200) pole figure. In 
this procedure, a tolerance $\varepsilon$ must be admitted which is slightly larger than the experimental uncertainty $\eta$ which in turn is composed of the natural peak width $\Delta \mathbf{y}$ of the pole figure peaks and the experimental resolving power $\Delta_{\exp }$ of the pole figure measurement.

The procedure was tested with several sets of randomly chosen orientations, the theoretical poles of which were shifted at random by an uncertainty $\eta \leq 1^{\circ}$. With these assumptions, $N=50$ orientations can be safely resolved. A value of $N=100$ represents the limit of resolving power.

The resolving power of the method can be increased by taking further pole figures into account. An extreme increase of resolving power can be achieved by decreasing the experimental uncertainty $\eta$ of the peak positions. This is possible in the case of individual crystallites of a multicrystal having natural peak widths in the range of $0.01^{\circ}$ if one uses an experimental method with a resolving power of the same order of magnitude. This can be achieved, for instance, by using synchrotron radiation. In such a case it should be possible to distinguish as many as $10^{7}$ individual orientations.

\section{ACKNOWLEDGEMENTS}

This work has been supported by the Deutsche Forschungsgemeinschaft.

\section{References}

Bunge, H. J. (1969). Mathematische Methoden der Texturanalyse. Akademie Verlag Berlin.

Bunge, H. J. (1982). Texture Analysis in Material Science. Butterworth Publ. London.

Bunge, H. J. and Esling, C. (1979). Journ. de Physique Lettres 40, 627-628.

Bunge, H. J. and Esling, C. (1985). Acta Cryst. A41, 59-67.

Bunge, H. J., Esling, C. and Muller, J. (1981). Acta Cryst. A37, 889-899.

Dahms, M. and Bunge, H. J. (1988). Textures and Microstructure 10, 21-35.

Matthies, S. (1979). Phys. Stat. Sol. (b) 92K, 135-138.

Maurer, R. and Gleiter, H. (1986). In: Experimental Methods of Texture Analysis. Ed. H. J. Bunge DGM Informationsgesellschaft Oberusel 347-357. 